\title{
El liderazgo educativo y el desempeño docente: un estudio etnometodológico de la realidad ecuatoriana
}

\section{Educational leadership and teaching performance: an ethnomethodological study of the Ecuadorian reality}

\author{
Vanessa Aracelly Del Salto Zurita ${ }^{1}$, Ana Isabel Fernández Lara ${ }^{2}$, Mónica del Carmen \\ Pachar Puga ${ }^{3}$
}

\section{Recibido: 18-10-2018 / Revisado: 28-11-2018 /Aceptado: 15-12-2018/ Publicado: 05-01-2019}

\begin{abstract}
.
DOI: https://doi.org/10.33262/cienciadigital.v3i1.287

Educational leadership and its impact on the teaching performance is the result of the need to solve a problem currently facing the education sector, for this reason, this work has been designed to train and guide teachers in his leadership capacity within the daily exercise. For the development of this research we observed the techniques of data collection based on contextualization in the educational field, allowing to define three categories that make up the field of representation: a) educational leadership; b) teacher performance and c) objectives, through which it is intended to detect or evidence a large number of processes in the performance of the teaching leadership that may be: ineffective, erroneous, ambiguous, inadequate or incongruent within the educational institution and in this way provide strategies to correct and solve them).
\end{abstract}

Keywords: Educational leadership, teaching performance, education, research, needs.

\footnotetext{
${ }^{1}$ Universidad Indoamérica, Facultad de Ciencias Humanas, de la Educación y Desarrollo social, Ambato, Ecuador, chely1917@live.com

${ }^{2}$ Universidad Indoamérica, Facultad de Ciencias Humanas, de la Educación y Desarrollo social, Ambato, Ecuador, anafernandez@uti.edu.ec

${ }^{3}$ Universidad Indoamérica, Facultad de Ciencias Humanas, de la Educación y Desarrollo social, Ambato, Ecuador, mpacharpuga@ hotmail.com
} 


\section{Resumen.}

La presente investigación es el resultado de la necesidad de dar solución a una problemática que actualmente atraviesa el sector educativo, por esta razón, el presente trabajo ha sido diseñado con el fin de capacitar y guiar a los docentes en su capacidad de liderazgo dentro del ejercicio diario.

Para el desarrollo de esta investigación se observaron las técnicas de recolección de datos partir de la contextualización en el ámbito educativo, permitiendo definir tres categorías que conforman el campo de representación: a) liderazgo educativo; b) desempeño docente $\mathrm{y}, \mathrm{c}$ ) objetivos planteados, a través de las cuales se pretende hallar o evidenciar una gran cantidad de procesos en el desempeño del liderazgo docente que pueden ser: ineficaces, errados, ambiguos, inadecuados o incongruentes dentro de la institución educativa y de esta manera proporcionar estrategias para corregirlos y solucionarlos.

Palabras claves: liderazgo educativo, desempeño docente, educación, investigación, necesidades.

\section{Introducción.}

El liderazgo es un proceso que se encuentra presente en todos los ámbitos de la sociedad, en el cual se encuentran inmersos hombres y mujeres denominados líderes que poseen una serie de características determinadas y desarrollan habilidades para guiar e influir a un grupo mediante la toma de decisiones acertadas, que benefician y motivan a su equipo u organización, con el objetivo de que todos trabajen con entusiasmo enfocados en alcanzar una meta en común, según Martí citado en Furguerle \& Vitorá, 2016, se puede decir también que "el liderazgo es la habilidad para ganar y retener seguidores, significa que solo es posible entenderlo desde la interacción, así como la influencia trasformadora ejercida sobre otros" de esta forma se puede entender que dentro del liderazgo existe un vínculo entre el líder y su grupo, mismo que le permite conocer la realidad del medio en el que se desenvuelven y poder realizar acciones concretas al momento de tomar decisiones, buscando el beneficio mutuo y un eficiente desempeño por parte del grupo que preside.

La educación actualmente atraviesa por una serie de cambios y trasformaciones orientados a obtener la excelencia académica, cada vez existen más estudios que hablan de la importancia del liderazgo docente, la exigencia de una calidad educativa, destaca la necesidad de vincular al sistema educativo a docentes con un perfil de líderes, que aporten de manera eficiente, integradora y creativa a los procesos de la educación, docentes con verdaderas habilidades de un líder capaz de conciliar y guiar a la comunidad educativa a llevar a cabo propuestas innovadoras que los conduzcan a conseguir sus metas deseadas.

Grandes organizaciones mundiales como la OREALC/UNESCO han dedicado mucho tiempo a realizar investigaciones en torno al liderazgo docente, ya que ellos consideran que 
"los líderes escolares son un verdadero tesoro para la educación” (Rojas \& Gaspar, 2006) como titula su publicación del libro sobre las bases del liderazgo en educación.

En Ecuador, el Ministerio de Educación ha venido implantando reformas en el sistema educativo, relacionadas con el desempeño de los directivos y docentes, como lo menciona la LOEI (2016) en su capítulo IV de la evaluación educativa Art.19 literales 2-3. Además, la ley orgánica de educación intercultural (LOEI) en su capítulo IV de la evaluación educativa Art.19 literales 2,3 menciona que se evaluara el desempeño de profesionales de la educación. Esto incluye el desempeño de docentes y de autoridades educativas y directivos (rectores, vicerrectores, directores, subdirectores, inspectores, subinspectores y otras autoridades de establecimientos educativos); y la gestión de establecimientos educativos, que incluye la evaluación de la gestión escolar de instituciones públicas fiscomisionales y particulares.

Además, el Instituto Nacional de Evaluación Educativa evalúa el desempeño institucional de los establecimientos educativos con un índice de calidad global que establece la ponderación de los diferentes criterios que miden la calidad educativa, elaborado por el Nivel Central de la Autoridad Educativa Nacional.

La ley Orgánica de Servicio Público LOSEP capítulo VII del subsistema de evaluación del desempeño a los funcionarios en su Art. 219 literal D que habla de la perspectiva del talento humano: que se incluirá en la evaluación del desempeño de la o el servidor la calificación de la calidad y resultados del liderazgo de los procesos internos, el uso eficaz del tiempo y la colaboración del trabajo en equipo de las y los servidores.

Vaillant, refiere en su artículo que "Cada ministerio e institución aúnan esfuerzos aplicando políticas y estrategias tratando de efectivizar la calidad educativa, pero pese a todo el trabajo de las autoridades gubernamentales y la comunidad educativa, aún sigue siendo un reto llegar obtener los resultados esperados" (2015).

Coral, G (2012) expresa que; la gestión educativa se complementa en todos los aspectos con el liderazgo que se ejerce dentro de una organización, pues se admite ampliamente cuyo ejercicio es un elemento clave en la mejora de lo que acontece dentro de las instituciones educativas.

En la Unidad Educativa Luis Chiriboga de la comunidad de Calhua Grande, parroquia Martínez de la ciudad de Ambato, se evidencian varios inconvenientes en la gestión que vienen realizando los docentes, autoridades y la comunidad educativa del sector, ya que la mentalidad de los padres de familia influye mucho sobre los docentes. Además, los estudiantes no poseen la motivación correcta y tienen escaso interés en aceptar la guía de los maestros. Por su parte, las autoridades institucionales encargadas han ejercido un tipo de liderazgo que difícilmente ha llegado a impactar positivamente en el desempeño de los docentes, por lo cual existen varias deficiencias dentro del grupo, que han llevado a los docentes a preguntarse en que si ellos ¿están ejerciendo un correcto liderazgo? Esta 
interrogante advierte la necesidad de los docentes de autoevaluarse, buscando una solución que les permita resolver la deficiencia en la gestión que realizan. El liderazgo siempre está presente en todo la realidad observada como lo cita: Argos, J. \& Asquerra, P. (2014). La temática del liderazgo se encuentra omnipresente en el contexto cotidiano, apareciendo por doquier, ya sea como causa o consecuencia de estados de cosas que se ocupan y/o preocupan; de situaciones a las que se enfrenta esta investigación y se desearía mantener, potenciar o trasformar, de realidades que se vislumbran como retos y cuya presencia es necesaria. Está claro la temática o problemática del liderazgo es algo constatable y apropiadamente vigente. Por otro lado, otro de los problemas que se evidencian es que las Autoridades suelen estar sobre exigidas a nivel administrativo y no tienen tiempo para tareas de índole más pedagógica por esta razón, participan poco de los procesos decisorios lo cual se convierte en una de las principales dificultades para consolidar un liderazgo efectivo, las exigencias externas han llevado en muchos casos, a una fragmentación de sus funciones. Vaillant, D. (2015).

Las reflexiones planteadas anteriormente, permiten formular el siguiente cuestionamiento que servirá de guia en el presente estudio ¿Los docentes de la Unidad Educativa Luis Chiriboga de la comunidad Calhua Grande se encuentran ejerciendo verdadero liderazgo en los procesos dentro de la Institución educativa?

La respuesta a esta interrogante es que no se están cumpliendo los procesos educativos eficientemente ya que se evidencia: la toma de decisiones ineficaces, escaso trabajo en equipo, dificultad para aplicar las reglas y normas de trabajo y convivencia, una mentalidad cerrada al cambio. Por tal motivo, es preciso llegar a la conciencia de los docentes, incentivándoles y capacitándoles como verdaderos líderes, conscientes de la realidad del entorno educativo, con mística en su trabajo, con vocación de servicio y comprometidos en poder llegar a una trasformación social. La presente investigación pretende aportar con la guía de un correcto liderazgo docente, que proporcione soluciones inmediatas y mejore el desempeño de los docentes de la unidad, guiándoles e incentivándoles a convertirse en verdaderos líderes educativos que incluyan la organización de programas, que no solo provean conocimientos y habilidades necesarios para funcionar efectivamente $y$ eficientemente, sino que también tengan una visión, una serie de actitudes, una variedad de perspectivas que provean una sólida base para cambiar y trasformar las formas en las cuales la escolarización, las políticas educativas, los currículos, la enseñanza y el proceso de aprendizaje han constituido su diario vivir. (Balda, R. \& Guzmán, A. 2015).

\section{Metodologia.}

Esta investigación tiene un enfoque cualitativo, ya que se lo realizó mediante una observación directa de la problematización en la institución educativa, posteriormente se lo baso en una revisión bibliográfica, ya que la información obtenida ha sido tomada de bases de datos y fuentes veraces y confiables como Redalyc, Google Académico y repositorios de Universidades Ecuatorianas y extranjeras, ya que estas aportan con la mayor cantidad de 
información con relación al Liderazgo y el desempeño del docente, en los cuales se pueden evidenciar que se ha realizado varios estudios que pretendiendo concientizar sobre el panorama que se vive entre la instituciones Educativas en América Latina y la importancia del ejercicio del liderazgo en el desempeño docente, como una fuente de oportunidades para lograr la excelencia académica y el éxito en la gestión educativa, los repositorios Universitarios de Ecuador permitieron tener una perspectiva de que porcentaje de investigaciones con relación a la temática existen en el país y la relevancia que tiene a nivel educativo y así poder enfocarnos en los resultados que se espera obtener a través de la aplicación de las guías y capacitaciones sobre liderazgo docente en la Institución educativa.

Para obtener un conocimiento más profundo de la temática planteada, es necesario tener una fundamentación teórica que parte desde la historia del liderazgo. El papel que desempeña un líder, los tipos de liderazgos, la contextualización del liderazgo a nivel educativo, el desempeño docente y posteriormente el propósito de conseguir una excelencia en la gestión educativa del Ecuador.

\section{Resultados.}

1. Historia del Liderazgo. Desde el inicio de la humanidad, aparece plasmado el concepto de liderazgo en la sociedad. Este ha sufrido varias trasformaciones, según los cambios, la evolución y el desarrollo humano a través del tiempo. La historia ha registrado la figura del líder como el encargado de guiar al pueblo, comunidad o la nación que presidía. Se consideraba al liderazgo desde un aspecto divino ya que los primeros habitantes consideraban que los dioses eran quienes enviaban al individuo indicado para guiarlos y orientarlos. Por mucho tiempo se consideraba como ley incluso la palabra del líder, pues lo consideraban alguien con mucha sabiduría, se lo veía incluso como una divinidad encarnada en un hombre, pues su capacidad de influencia entre los habitantes era muy alta.

Con el paso del tiempo y a medida que los reinos se expandían y fortalecían, el liderazgo también lo hacía, y con esto los líderes evolucionaban y mejoraban sus formas de administrar a la sociedad. (Giraldo \& Naranjo, 2014).

Aparecían nuevos Hombres con capacidades impresionantes para influenciar y liderar a las multitudes, muchos de ellos aún son recordados como una fuente de inspiración, de los cuales se puede mencionar a; Jesucristo, Napoleón Bonaparte, Julio Cesar, Martin Lutherking Mahatma Gandhi, Nelson Mandela y el mismo Libertador Simón Bolívar, quienes marcaron un precedente sobre el liderazgo, invitándonos a reflexionar sobre la forma que tiene un verdadero liderazgo de impacto en la sociedad.

Desde entonces y a raíz de esto, el liderazgo se fue convirtiendo en uno de los temas de estudio de muchos filósofos y sabios de todas las épocas, los cuales empezaron a escribir manuales y libros sobre el tema, analizando desde el ámbito administrativo y organizacional hasta el ámbito militar. (Giraldo \& Naranjo, 2014). 
Algunos de los aportes citados por Estrada (en Giraldo \& Naranjo, 2014): "Confucio en el siglo VI A. C. escribió un manual sobre su gobierno y administración y al cual se le dio características de Constitución, que estableció reglas sobre organización, funciones, procedimiento, labores de rutina, controles, castigos y registros”. Sun Tzu escribió el Arte de la Guerra, "el tratado militar más antiguo del mundo, que aún está en vigencia. Este libro es una valiosa guía para los jefes o líderes militares y administradores ya que posee consideraciones importantes en relación con la planeación, la organización y la dirección." (p12).

En Grecia se discutieron y analizaron los conceptos de origen y actualización del Estado, lo cual permitió el desarrollo de un gobierno democrático, similar al actual. Adicionalmente, en esta civilización se encuentra el origen del método científico que influyó en la administración, como se reflejan los estudios de Frederic W. Taylor, Frak B. Gilbreth, Henri Fayol y otros.

De esta manera, se puede determinar que aun los estudios que fueron realizados en torno al liderazgo buscaban definir las estructuras sobre cómo se podría dirigir a la sociedad y al crear un análisis de los grandes líderes de la historia del mundo, podrían identificar cuáles eran esas características que los diferenciaban de los demás. (Giraldo \& Naranjo, 2014), y así poder tener una referencia del liderazgo que sirva como una guía eficaz que pueda ser aplicada.

En estudios realizados más actualmente (Rojas \& Gaspar, 2006) definen que: “el liderazgo es el arte de la conducción de seres humanos" Y que "Por lo tanto, los/las líderes son personas o grupos de personas son competentes en el arte de conducir a una comunidad en la construcción de un futuro deseable por/para esa comunidad" que presiden.

Chiavenato (citado en Furguerle R, J. \& Vitorá, M, 2016) lo plantea como "un fenómeno social, por ello se concibe como la influencia interpersonal ejercida en determinada situación para la concreción de uno o más objetivos". Todos estos conceptos sirven de base para poder definir que el liderazgo es un proceso sumamente valioso que se encuentra vigente en la actualidad y que está orientado a influenciar, orientar, guiar y dirigir el entorno que los rodea.

2. Papel que desempeña el líder. Para Gómez, (2006) expresa que: Líder es un individuo capaz de ejercer influencia en otros, para dirigirlos y guiarlos efectivamente hacia el logro de objetivos y metas organizacionales.

Por su parte el libro publicado por OREALC/UNESCO (2006) dice que los/las líderes son personas (o conjuntos de personas) competentes en el arte de conducir hacia el futuro. Es decir, que los y las líderes y lideresas están plenamente facultados para dirigir a sus grupos hacia el futuro o la meta a conseguir. 
Otros criterios pueden definir al líder como el conductor de grupo, como un orientador y un dirigente de la dinámica grupal que coadyuva a conseguir con eficacia y certeza las metas y objetivos que se plantea un grupo, como un referente o guía que impulsa a sus seguidores todo el tiempo.

Ahora cabe recalcar que no siempre el directivo que está al frente de alguna empresa o institución, y que posee la mayor jerarquía, es necesariamente un líder, puede ocupar solo el cargo de jefe y aun así no ejercer liderazgo dentro del grupo, existe una gran diferencia entre ser líder y jefe muchas veces se puede llegar a pensar que al estar en una organización con una estructura jerárquica bien definida el líder única y exclusivamente es la persona de rango superior. (Giraldo \& Naranjo, 2014).

Pero no es así, por eso es necesario determinar cuál es la diferencia entre el jefe y el líder; el jefe es toda aquella persona de rango superior que lo único que hace es asignar funciones para gestionar su organización, esperando que toda persona de rango inferior (subordinado) cumpla cabalmente con lo que se le ordena. Por otro lado, si bien en la mayoría de casos el líder es una persona de rango superior liderazgo proactividad, creatividad, influencia trabajo en equipo, seguidores, proyectos y resultados conjuntos, comunicación asertiva, no significa que siempre sea así, por el contrario, un líder puede ser cualquier persona de cualquier rango que fomente el trabajo en equipo, interactuando, estimulando e influyendo a sus seguidores al cumplimiento de metas y objetivos que comparten, como menciona Gómez \& Hernández, citado en Giraldo \& Naranjo, (2014).

El líder es quien guía, orienta e impulsa al grupo a seguir la línea decisoria por medio de su interacción e influencia sobre un grupo de personas (seguidores). Este, se vale de sus cualidades como la comunicación asertiva, la creatividad, la proactividad y el trabajo en equipo, para lograr las metas y objetivos que comparte con sus seguidores. Es capaz de influir de manera positiva o negativa al conducir su grupo al éxito total o en complemento a la ruina, (Gómez, 2008).

De esta manera se puede determinar que la figura del líder es sumamente importante, el papel del líder va más allá de un jefe. Ser líder implica una gran responsabilidad, ya que cada decisión tomada, afectará directamente al grupo que dirige. De este entramado de particularidades dependerá el futuro de la empresa, organización o institución educativa.

3. Contextualización del liderazgo a nivel educativo. A nivel educativo, el liderazgo también desempeña un papel fundamental, ya que es primordial que las autoridades, los directivos y los docentes, se conviertan en verdaderos líderes, capaces de guiar a todos los individuos que conforman la comunidad educativa a lograr éxito en la gestión de los procesos educativos.

Basándose en estas necesidades, en los últimos años se han creado varias investigaciones como lo mencionan: Scribner, Sawyer, Watson, \& Myers (2012) donde expresan que: "las investigaciones que se han generado en torno al liderazgo en los contextos educativos han 
surgido como una necesidad de dar respuesta al fracaso de muchas políticas educativas en el ámbito nacional que no han podido insertarse exitosamente en la realidad local". Por su parte, Vaillant, D. (2015) menciona que "Las investigaciones entorno los temas de liderazgo se han multiplicado y se han centrado en el análisis de la figura del líder y también en sus funciones y tareas", buscando que tales investigaciones sirvan de aporte al sistema educativo.

Liderazgo Directivo, Furguerle, J. \& Vitorá, definen claramente la idea exponiendo: El liderazgo del directivo es una acción a través del cual se hace posible el logro de los objetivos de la institución, mediante un proceso dirigido a racionalizar el talento humano; así como los recursos técnicos y materiales con los fines de productividad. Por lo tanto, las nuevas teorías, desde la perspectiva de la administración, conciben al liderazgo a partir de la eficacia; es decir, la capacidad de respuesta que los aparatos educativos brindan ante las exigencias de parte de la comunidad.

El principal tipo de liderazgo que se ejerce en las Instituciones Educativas es el liderazgo Directivo; ya que el directivo en las instituciones educativas reúne un conjunto de características personales y habilidades de gestión, una de las principales características del directivo que el directivo como líder puede desarrollar se encuentra en el ser; es decir, en la práctica y en la vivencia de los valores, las cuales le permiten lograr con éxito las metas con la participación activa de los actores educativos.

El liderazgo Directivo en las Instituciones es de suma importancia ya que el directivo en su papel de líder motiva y está en la capacidad de generar estrategias para fomentar la motivación extrínseca. Con ello se fomentan aspectos positivos en el comportamiento de los docentes con respecto a la actividad ejecutada y, entonces, resulta importante el incentivo externo en la búsqueda de alcanzar los objetivos, en procura de elevar la calidad académica, organizativa del plantel (2016).

Al hablar de motivación se menciona también al manejo de las emociones, lo cual es una herramienta útil por parte del directivo ya que el liderazgo se prueba en situaciones complejas, en momentos difíciles o críticos, es ahí donde se ponen en juego la capacidad del líder para tomar control de la situación que se presenta por ejemplo: dependiendo del lugar en donde está ubicada la Institución educativa suelen presentarse conflictos de diversa índole, pueden ser familiar, derivada de las tensiones sociales o de fenómenos como el narcotráfico. Ante ello, directivos y maestros deben lograr cumplir no solamente con el cien por ciento de lo exigible, sino ir más allá y entregar más tiempo y sus mejores esfuerzos. Tal cosa no es posible sin una alta motivación de todo el plantel. El liderazgo trata justamente de eso, de cómo lograr que una comunidad humana, una escuela, una empresa, una comuna o municipio, una nación, entregue sus mayores esfuerzos, no por coerción sino por convicción propia. Lograr eso requiere motivación y la motivación es asunto de liderazgo, OREALC/UNESCO (2006). 
Aplica normas de comunicación asertiva con el grupo de docentes, con el propósito de que a través de la aplicación de la comunicación también influye en las opiniones, actitudes o conductas de los miembros internos y externos de la institución, a fin de garantizar el cumplimiento de los objetivos. Por ello, es necesario contar con técnicas o actividades de investigación, pues a través de ella se conocerán los problemas, necesidades y áreas de oportunidad en materia de comunicación.

Es un ente dispuesto al cambio que conduce al grupo a mirar hacia alternativas de innovación, creatividad y actualización, ya que siempre está dispuesto aprender cosas nuevas. Además, este permanece capacitándose continuamente consiente que en materia de educación y ciencia, siempre existirán nuevos avances, que exigen el permanecer actualizándose constantemente. Mijares citado por Furguerle, J. \& Vitorá, M, menciona lo siguiente: en la naturaleza, en el desarrollo humano y en la inventiva del hombre nada es estático. Este concepto necesita ser aplicado en todo cuanto se hace, por tanto, el desarrollo emocional e intelectual no se da espontáneamente, como el biológico, y por ello se necesita asumir la importancia del cambio el diario que hacer. Ante esta perspectiva, se necesita estar consciente de que siempre va a existir oposición al cambio, porque da temor lo nuevo y saca a la persona de la zona de seguridad (2016)

\section{Liderazgo Docente.}

En un informe de UNESCO Vaillant, D, señala que uno de los aspectos importantes de la mejora del proceso de aprendizaje es el énfasis en el apoyo y presión positiva de los líderes escolares hacia los estudiantes.

Por lo general el liderazgo se lo atribuye a los directivos de las instituciones educativas e incluso la mayor parte de investigaciones que se hacen son dirigidas hacia determinar cuáles deberían ser las características o el estilo de liderazgo del personal directivo. Es importante señalar que el trabajo que puede realizar el liderazgo docente es aún más efectivo ya que el docente, maneja las mismas características que un líder directivo porque las desarrolla de manera más directa, aplica la motivación, impulsa el trabajo en equipo, despierta un compromiso en los estudiantes, es innovador y defiende la innovación en los procesos, mejora la calidad educativa, reduce y resuelve conflictos, fomenta mejores relaciones interpersonales con sus alumnos y sus compañeros docentes, actúa como un ente conciliador en situaciones de tensión guiando a que la institución o el lugar donde él trabaja sea un ambiente de paz. Además, una de las características más pertinentes se orienta a la capacidad de liderazgo, que incide en el aprendizaje de los estudiantes. Citando el informe de UNESCO se señalan los aspectos importantes de la mejora del proceso de aprendizaje, a saber: el énfasis en el apoyo y presión positiva de los líderes escolares hacia los estudiantes. UNESCOIIPE-IWGE , (2015). 
Robinson afirma lo citado de Vaillant, D, La tendencia actual insiste en que éxito del liderazgo escolar está en la incidencia en los aprendizajes de los alumnos a través de la mediación de los directores y docentes. Por esa razón, la literatura crecientemente afirma que los directores deben favorecer la mejora del desarrollo profesional de maestros y profesores para incrementar así los resultados de los alumnos. La buena gestión del director por sí sola es insuficiente, también debe posibilitar buenos aprendizajes de los estudiantes. Por esa razón, para evaluar la eficacia del liderazgo educativo hay que considerar el impacto en el aprendizaje y resultados de los alumnos como lo expresa (2015).

En un análisis realizado por Bolivar donde plantea lo siguiente: De manera que el liderazgo no depende de una cúspide o primera cadena de mando. Bien entendido, en una organización que se «mueva», debe estar ampliamente compartido, de modo que los docentes, al trabajar conjuntamente, desarrollan competencias y ejercen influencia mutua, Acentuar la dimensión de compartición en el liderazgo educativo responde a un modo de entender que la enseñanza y el aprendizaje han de ser el foco principal de la escuela, que los directivos no pueden soportar todo el peso de la dirección del centro docente debido a las crecientes presiones y exigencias que se imponen a sí mismos y a las escuelas, dimensiones que han sido subvaloradas.

Todos estos aspectos contribuyen a comprender que no se necesita ser autoridad educativa para ejercer liderazgo. En contraste, este puede ser compartido con todos los docentes y se generaran mejores resultados obtenidos de la gestión. Existe una conexión entre el incremento de papeles y responsabilidades de liderazgo con la mejora de resultados de los alumnos. La investigación ha evidenciado que el liderazgo escolar tiene una influencia mayor en las escuelas y en los estudiantes cuando se encuentra ampliamente distribuido (2011).

El desempeño docente. Es necesario hablar de esta temática, ya que la presente investigación pretende determinar la incidencia que tiene el Liderazgo en el desempeño docente. Se parte de esta premisa para afirmar que el desempeño docente está relacionado a un conjunto de acciones organizadas de acuerdo con las mediaciones e interacciones pedagógicas entre el conocimiento científico y el conocimiento escolar, y con las mediaciones socioculturales y lingüísticas.

El desempeño docente generalmente es evaluado de acuerdo con las normas de calidad educativa de cada país, pero ¿Qué son los patrones de calidad? Pues es sencillo los estándares de calidad son un conjunto de parámetros e indicadores bajo los cuales es medido o evaluado el docente, en Ecuador se considera que las normas de desempeño docente son descripciones de lo que debe hacer un profesor competente; es decir, de las prácticas pedagógicas que tienen más correlación positiva con el aprendizaje de los estudiantes. MINEDUC, (2011).

La SEP (citada en Martínez, Guadalupe, \& Guevara, 2015), menciona que la evaluación del desempeño docente es un proceso sistemático cuyo propósito es emitir juicios de valor sobre 
la calidad del cumplimiento de responsabilidades docentes en la enseñanza, aprendizaje y desarrollo de los estudiantes, a través de un seguimiento permanente.

Excelencia en la gestión educativa del Ecuador. La constitución política del Ecuador establece en su artículo 26 que "la educación es un derecho de las personas a lo largo de su vida y un deber ineludible e inexcusable del Estado", y en su artículo 27 agrega que la educación debe ser de calidad. Adicionalmente, la sexta política del Plan Decenal de Educación determina que hasta el año 2015, se deberá mejorar la calidad y equidad de la educación e implementar un sistema nacional de evaluación y rendición social de cuentas del sistema educativo, pese a que estos artículos se encuentran plasmados como mandatos, no expresan directamente lo que significa la calidad educativa, por lo cual se analizará a continuación:

Para establecer qué es una educación de calidad, se requiere identificar qué tipo de sociedad se debe tener, pues un sistema educativo será de calidad en la medida en que contribuya a la consecución de esa meta. Por ejemplo, para ser conducente a una sociedad democrática, el sistema educativo será de calidad si desarrolla en los estudiantes las competencias necesarias para ejercer una ciudadanía responsable. En el caso ecuatoriano, según señala la Constitución, se busca avanzar hacia una sociedad democrática, soberana, justa, incluyente, intercultural, plurinacional y segura. Con personas libres, autónomas, solidarias, creativas, equilibradas, honestas, trabajadoras y responsables, que antepongan el bien común al bien individual. Que vivan en armonía con los demás y con la naturaleza, y que resuelvan sus conflictos de manera pacífica.

Adicionalmente, un criterio clave para que exista calidad educativa es la equidad. Equidad en este caso se refiere a la igualdad de oportunidades, a la posibilidad real para el acceso de todas las personas a servicios educativos que garanticen aprendizajes necesarios, así como su permanencia y culminación en dichos servicios.

Por lo tanto, de manera general, el sistema educativo ecuatoriano será de calidad en la medida en que dé las mismas oportunidades a todos. Además, cuando los servicios que ofrece, los actores que lo impulsan y los resultados que genera contribuyan a alcanzar las metas conducentes al tipo de sociedad que se aspira para el país. MINEDUC, (2011).

Como se mencionó anteriormente, existen diversos informes internacionales (OCDE, 2013, UNESCO-IIPEIWGE, 2012) que identifican la función de liderazgo escolar como clave para asegurar la calidad de la educación. El Ministerio de Educación de Ecuador (Vaillant, 2015) respalda el interés en su lucha al insertar políticas públicas en busca de la calidad educativa. 


\section{Conclusiones.}

- Por mucho tiempo la carrera docente se ha visto desmerecida y relegada, los docentes en Ecuador como en otros países de América Latina, viven una realidad cotidiana de sobre exigencias externas a nivel administrativo, las entidades educativas como el ministerio y los distritos, se mantienen continuamente exigiendo a las Instituciones Educativas que cumplan con entregar Informes, matrices y planificaciones de las clases y por cada actividad que realizan, como proyectos y fiestas de lectura, los maestros debían completar al menos 40 documentos como parte de su tarea administrativa en el aula, posteriormente para compensar la carga y la exigencia se redujo a 18 documentos, pero literalmente la carga sigue siendo grande y un motivo de estrés en el gremio docente, ya que esta situación verdaderamente ha fragmentado la gestión de los lideres educativos, quienes no tienen espacio para pensar en capacitarse o analizar el tipo de liderazgo que están ejerciendo en las Instituciones, lo cual es muy preocupante si se piensa en obtener una calidad educativa.

- Otra situación que se puede apreciar, es el poco interés y el desconocimiento que tienen los docentes con relación al liderazgo, ya que ellos consideran que el liderazgo es un tema que solo debe interesar a las autoridades de la Institución, lo cual deja a los docentes caer en un pensamiento tradicionalista que no busca influir, guiar u orientar a los estudiantes, los docentes deben enseñar la parte teórica de sus asignaturas y cumplir con las exigencias administrativas, pero pierden recursos muy valiosos que el liderazgo puede aportar a su gestión educativa. Por lo tanto, su desempeño no es el más óptimo, ya que no se llega a la excelencia, sino al plano de cumplir con lo asignado sin importar la forma en la que se lo haga.

- Los directivos tienen más características de jefe que de líder, lo cual debería llevarlos a reflexionar sobre su gestión educativa, otro inconveniente es que muchas veces el liderazgo de la institución está monopolizado solo en la autoridad y no repartido entre todos los docentes, lo cual provoca un sobre cargo y la ineficiencia a la hora de cumplir con los procesos, y en ocasiones ni siquiera se alcanza a cumplir con todo lo solicitado.

- Si bien es cierto que el ministerio de educación programa capacitaciones para los docentes a través de la página mecapacito.com, es escaso encontrar cursos o capacitaciones en torno al liderazgo docente, aun cuando esta es una temática muy importante, no se la toma en cuenta como una herramienta útil que pueda guiar a los docentes a mejorar su desempeño dentro y fuera de las aulas.

- En el Ecuador es escaso encontrar investigaciones o documentación que hablen del tema, es cierto que los documentos oficiales del ministerio de educación, la constitución y en el plan decenal, se encuentra prevista la temática, pero en el contexto real no es aplicada, o por lo menos no tiene injerencia al momento de realizar la gestión educativa. 
- La importancia del liderazgo en las instituciones educativas, debe concientizar a los países a reformar sus procesos educativos y así poder obtener la excelencia académica, como lo mencionan los Informes emitidos en los últimos años por; OCDE, UNESCO-IIPEIWGE, citado de Vaillant, los cuales "Identifican la función de liderazgo escolar como clave para asegurar la calidad de la educación”.

\section{Referencias bibliográficas.}

Argos, J. \& Asquerra, P. (2014) en su libro de Liderazgo y educación. Recuperado de google books: https://books.google.es/books?id=MYZPBQAAQBAJ\&printsec=frontcover\&hl=es $\# \mathrm{v}=$ onepage $\& \mathrm{q} \& \mathrm{f}=$ false.

Ahumada, L. (2012) Liderazgo en organizaciones educativas. núm. 15, enero-diciembre, 2012, pp. 239-252.Recuperado de http://www.redalyc.org/articulo.oa?id=147125259014

Balda, R. \& Guzmán, A. (2015). Liderazgo transformacional como necesidad de las instituciones educativas. Vol. 3, Año 2015, No. 2.Recuperado de file:///C:/Users/HP/Downloads/370-906-3-PB.pdf

Bolívar, A. (2011) Aprender a liderar líderes. Competencias para un liderazgo directivo que promueva el liderazgo docente, Universitat Autónoma de Barcelona, Barcelona, España

Coral, G. (2012). Gestión del liderazgo y valores en la Escuela de práctica Docente Leopoldo, N. Chávez, (tesis de posgrado inédita), Universidad Técnica Particular de Loja \& La Pontificia Universidad Católica Del Ecuador Pichincha, Quito.

Gómez, R. (2006). El liderazgo empresarial para la innovación tecnológica en las micro,pequeñas y medianas empresas . Bogotá-Colombia : Universidad del Rosario .

Furguerle R, J. \& Vitorá, M.(2016) Liderazgo en los directivos de educación primaria Telos, vol. 18, núm. 2, mayo-agosto, 2016, pp. 208-227.Recuperado de http://www.redalyc.org/articulo.oa?id=99345727004

Martínez, C., Guadalupe, I.\& Guevara, A.(2015) La evaluación del desempeño docente, vol. 11, núm. 4, julio-diciembre, 2015, pp. 113-124 Recuperado de

http://www.redalyc.org/articulo.oa?id=46142596007

OREALC/UNESCO Santiago, Libro Bases del liderazgo en educación, Impreso en Chile por Andros Impresores Santiago, Chile; agosto, 2006

http://unesdoc.unesco.org/images/0014/001470/147055s.pdf 
Giraldo, D. \& Naranjo, J. (2014) Liderazgo: desarrollo del concepto, evolución y tendencias (estudio monográfico inédito) Universidad Del Rosario, Colombia, Bogotá D.C.

Gómez, R. (2006) El liderazgo empresarial para la innovación tecnológica en las micro, pequeñas y medianas empresas, Universidad del Rosario Bogota, Colombia

Gómez, R.(2008) El liderazgo empresarial para la innovación tecnológica en las micro, pequeñas y medianas empresas. Pensamiento y Gestión, 24, 157-194.

MINEDUC, (2011) Estándares de Desempeño Profesional Docente, Quito - Ecuador.

La ley orgánica de educación intercultural LOEI (2016)

Reglamento general a la ley orgánica del servicio público R-LOSEP (2016)

Vaillant, D. (2015).) Documento de antecedentes preparado para el Informe de seguimiento de la educación para todos en el mundo 2015. Educación para todos 2000-2015: logros y desafíos Liderazgo escolar, evolución de políticas y prácticas y mejora de la calidad educativa (Informe ED/EFA/MRT/2015/PI/9). Recuperado de http://unesdoc.unesco.org/images/0023/002324/232403s.pdf

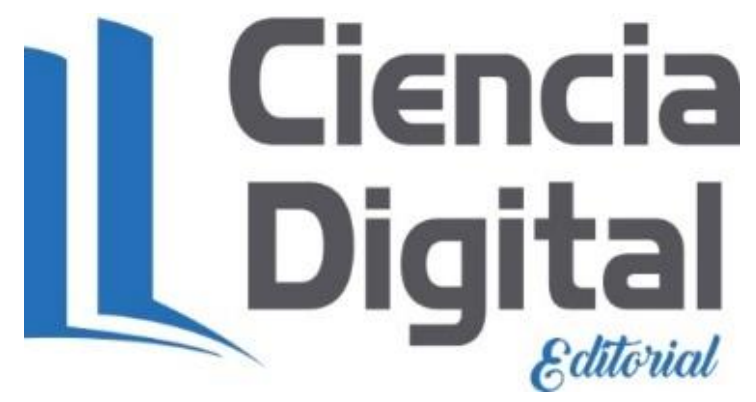




\section{Para citar el artículo indexado.}

Del Salto V, Fernández A, \& Pachar M. (2019). El liderazgo educativo y el desempeño docente: un estudio etnometodológico de la realidad ecuatoriana. Revista electrónica Ciencia Digital 3(1), 257-271. Recuperado desde: http://cienciadigital.org/revistacienciadigital2/index.php/CienciaDigital/article/view/287/68 $\underline{8}$

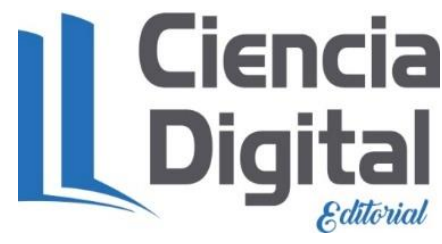

El artículo que se publica es de exclusiva responsabilidad de los autores y no necesariamente reflejan el pensamiento de la Revista Ciencia Digital.

El artículo queda en propiedad de la revista y, por tanto, su publicación parcial y/o total en otro medio tiene que ser autorizado por el director de la Revista Ciencia Digital.
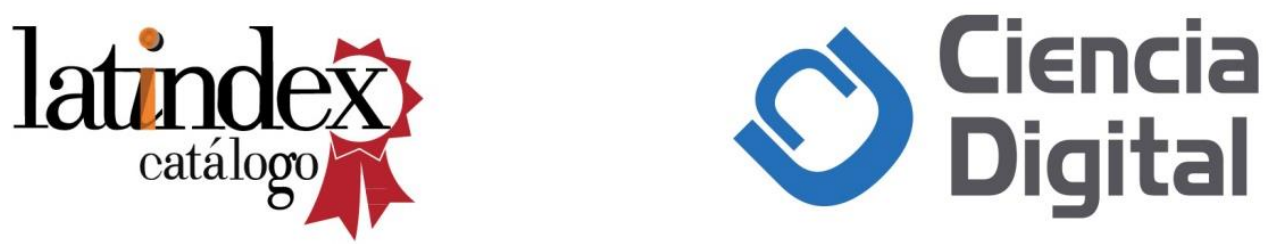